\title{
Aprendizagem do medo por observação em pombos (Columba livia)
}

\author{
Fear learning by observation in pigeons (Columba livia) \\ Aprendiendo el miedo a través de la observación en \\ palomas (Columba livia)
}

\author{
Andressa Pelaquim ${ }^{1}$, Fernando Bicocchi Canova², \\ Elenice Aparecida de Moraes Ferrari ${ }^{3}+$
}

1.Doutoranda, Universidade Estadual de Londrina, UEL. Londrina-PR, Brasil.

2.Professor Doutor, Universidade de Mogi das Cruzes, UMC. Mogi das Cruzes-SP, Brasil.

3.Professora Doutora, Universidade Estadual de Campinas, UNICAMP. Campinas-SP, Brasil.

†In Memmorian.

\begin{abstract}
Resumo
Introdução. Estudos com roedores e primatas demonstram que a aprendizagem do medo ocorre por experiência com contingências aversivas ou por observação da experiência de outros. Objetivo. Analisar a aquisição da resposta condicionada de medo por pombos que observaram o treino de condicionamento clássico som-choque em um animal modelo. Método. Pombos, machos, adultos, (Columba livia), atribuídos aos grupos: Grupo Modelo e Grupo Observação. O procedimento experimental foi conduzido com pombos treinados (modelo) e pombos observadores. Os grupos, foram expostos ao treino com três pareamentos somchoque, seguido pelo teste ao contexto $24 \mathrm{~h}$ depois e teste ao som, $48 \mathrm{~h}$ depois. As sessões foram gravadas para transcrição e análise de comportamentos. Os dados comportamentais foram analisados com o teste ANOVA e com o teste Tukey para comparações post hoc. Resultados. Os Grupos Modelo e Observação foram caracterizados por apresentação de congelamento, comportamento tipicamente estudado em trabalhos que envolvam aprendizagem aversiva. Verificou-se o mesmo padrão de comportamentos para os pombos Modelos e Observadores, o que indica que a experiência de observar outro pombo durante o treino aversivo resultou em comportamentos condicionados de medo, demonstrando aprendizagem de medo por observação em pombos. Conclusão. Esses dados corroboram com resultados obtidos em primatas e roedores
\end{abstract}

Unitermos. Postura; Condicionamento; Aprendizagem; Medo

\begin{abstract}
Introduction. Studies in rodents and primates have shown that fear learning occurs through experience with aversive contingencies or by watching the experience of others. Objective. To examine the acquisition of conditioned fear response by pigeons who observed the classical conditioning tone-shock training in an animal model. Method. Pigeons, adult male (Columba Livia), assigned to groups: Model Group and Observation Group. The experimental procedure was conducted with trained model pigeons and observers' pigeons. The groups were exposed to training with three pairings tone-shock, followed by the context test 24 hours later and the tone test, 48 hours later. The sessions were recorded for transcription and behavior analysis. Behavioral data was analyzed using ANOVA and Tukey test for post hoc comparisons. Results. Model Groups and Observation Groups were characterized by presentation of freezing behavior typically studied in work involving aversive learning. It was the same pattern of behavior for pigeons Models and Observers, which indicates that the experience of observing other pigeon during aversive training resulted in conditioned fear behaviors, demonstrating fear learning by observation in pigeons. Conclusion. These data corroborate results obtained in primates and rodents

Keywords. Conditioning; Learning; Fear
\end{abstract}




\section{Resumen}

Introducción. Los estudios con roedores y primates demuestran que aprender a temer ocurre a través de la experiencia con contingencias aversivas o mediante la observación de la experiencia de otros. Objetivo. Analizar la adquisición de la respuesta de miedo condicionada por palomas que observaron el entrenamiento clásico de condicionamiento de sonido-choque en un animal modelo. Método. Palomas, machos, adultos, (Columba livia), asignados a los grupos: Grupo Modelo y Grupo de Observación. El procedimiento experimental se realizó con palomas adiestradas (modelo) y palomas observadoras. Los grupos fueron expuestos a un entrenamiento con tres emparejamientos de choque de sonido, seguido de la prueba de contexto 24 horas después y la prueba de sonido 48 horas después. Las sesiones se registraron para análisis de transcripción y comportamiento, los datos de comportamiento se analizaron mediante la prueba ANOVA y la prueba de Tukey para comparaciones post hoc. Resultados. Los Grupos de Modelo y Observación se caracterizaron por congelar la presentación, un comportamiento típicamente estudiado en trabajos que involucran aprendizaje aversivo. El mismo patrón de comportamiento se encontró para las palomas modelo y observadoras, lo que indica que la experiencia de observar a otra paloma durante el entrenamiento aversivo resultó en un comportamiento condicionado por el miedo, lo que demuestra el aprendizaje del miedo mediante la observación en las palomas. Conclusión. Estos datos corroboran con los resultados obtenidos en primates y roedores

Palabras clave. Condicionamiento; Aprendizaje; Miedo

Trabalho realizado na Universidade Estadual de Londrina, UEL. Londrina-PR, Brasil.

\section{INTRODUÇÃO}

O Condicionamento clássico do medo ou simplesmente condicionamento aversivo é definido com a associação entre dois estímulos, um condicionado, como um som ou luz e um estímulo incondicionado, como um choque elétrico. A partir do pareamento desses estímulos, o primeiro adquire capacidade de eliciar respostas incondicionadas. No condicionamento ao contexto, o ambiente pode ser associado com um choque elétrico, passando a eliciar respostas aversivas ${ }^{1-3}$.

A interação social entre os indivíduos de um mesmo grupo garante a possibilidade de aprendizagem por 
observação ou por imitação do comportamento de outro coespecífico. Essa aprendizagem, geralmente classificada como aprendizagem social, tem um valor adaptativo importante por permitir que um indivíduo se beneficie da experiência de outros, aumentando, por exemplo, a eficiência na obtenção de alimentos, cuidados e proteção necessários à sobrevivênciaa-6.

Diferentes espécies de pássaros demonstram capacidade de aprender sobre a disponibilidade e de fontes de alimentos a partir da interação social. Em nosso laboratório, muitos estudos investigaram a capacidade de aprendizagem por observação social em pombos e pintainhos. Estes foram conduzidos pela análise de processos que contribuem para a formação de associações entre diferentes estímulos e entre respostas e estímulos, que são expressas posteriormente na ausência do animal da mesma espécie que serviu como modelo para aprendizagem ${ }^{7}$. Dada à função adaptativa dessa capacidade, não é surpreendente que muitos animais, incluindo pássaros, camundongos, gatos, vacas e primatas, possam aprender a ter medo pela observação de co-específicos.

A experiência de observar o comportamento de outro pombo numa situação de discriminação de pares de estímulos, facilita a aprendizagem da discriminação pelo observador $^{8}$. Pesquisadores relataram que os pombos que observaram outro pombo (pombo-modelo) durante a aprendizagem de como empurrar uma porta corrediça com o bico para ter acesso ao alimento, exibiram maior proporção 
de respostas de empurrar do que os pombos-controles ${ }^{9}$. Em estudos realizados com pombos, estes animais observaram um pombo-modelo, que estava numa câmara adjacente, durante a emissão de uma resposta de subir numa caixa e bicar o disco 10 vezes. Verificaram que os observadores adquiriram a resposta somente quando foram reforçados diferencialmente frente ao comportamento requerido do demonstrador, que serviu como estímulo discriminativo ${ }^{10}$.

Estudos com pintainhos (pintinhos) mostraram que eles se orientam e despenderam maior atenção para sítios ou tipos de alimentos que são direta ou indiretamente indicados pela galinha-mãe e, desse modo, são capazes de aprender a selecionar alimentos a partir da observação de outros ${ }^{4-6}$. Foi demonstrado ainda que, pintainhos com idade de um dia aprenderam por observação uma resposta de esquiva inibitória. Animais que observaram outro pintainho (modelo) bicar um substrato com contas que tinham gosto amargo (embebidas em methylanthranilate) e, por isso, exibiram respostas de repulsa (por exemplo, balançar violentamente a cabeça com o bico aberto), evitaram bicar contas da mesma cor que lhes foram apresentadas durante o teste ${ }^{11}$. Outros estudos confirmaram tais evidências e, ao mesmo tempo, estenderam a investigação para a análise desse tipo de aprendizagem de esquiva inibitória em pintainhos de substratos neurais, demonstrando a participação de estruturas dos dois hemisférios cerebrais, com um curso temporal de eventos neurais que indicam um papel crucial para o hemisfério direito ${ }^{12-14}$. 
Deve-se ainda destacar que no contexto da aprendizagem social, a aprendizagem do canto em pássaros demonstra a importância do ambiente social para a aquisição de padrões comportamentais adaptativos ${ }^{15-17}$. Embora 0 desenvolvimento do canto específico da espécie seja regulado pela maturação e pela variação hormonal sazonal, as variações regionais no canto de muitas espécies parecem depender da experiência com co-específicos ${ }^{18}$. Aves canoras são capazes de aprendizagem da comunicação vocal, constituindo, assim, modelos adequados para o estudo dos mecanismos neurais de processos sensoriais e motores complexos.

A exposição a ambientes aversivos e ameaçadores desencadeia respostas de defesa e resulta em formação da memória de experiências aversivas que persistem no tempo. Tais experiências podem envolver o condicionamento clássico aversivo com processos comportamentais complexos cuja organização envolve a atividade em circuitos neuronais que integram diferentes áreas cerebrais.

Num ambiente que está constantemente mudando é essencial que o indivíduo aprenda quais são os estímulos e eventos que são potencialmente perigosos. Situações potencialmente ameaçadoras ou perigosas levam ao desenvolvimento de estados emocionais e motivações relacionadas com os comportamentos de fuga, esquiva, medo e ansiedade, entre outros. Os comportamentos de medo podem ser expressos, transmitidos e adquiridos de 
várias maneiras, seja por experiência direta ou por meio de transmissão social ${ }^{8}$.

É fundamental que o indivíduo saiba reconhecer se uma situação é ameaçadora e qual tipo de perigos ela representa, e em humanos, essa capacidade envolve processos comportamentais e cognitivos, experiência pessoal e cultural, além de uma história evolutiva que inclui fontes de seleção natural que foram comuns para outras espécies. De fato, existe um grande conjunto de estudos em primatas e roedores que demonstram existir uma relação entre padrões comportamentais de defesa que se correlacionam com situações específicas de ameaça ${ }^{19}$.

A transmissão social e detecção de sinais de medo são bem documentadas em uma grande variedade de espécies, sendo que esses sinais não apenas alertam aqueles que os recebe sobre a potencialidade e iminência do perigo, mas também atribuem valores negativos ao contexto ou aos estímulos associados com a situação. Por exemplo, a expressão de medo de um co-específico pode servir como um estímulo incondicionado, eliciando uma resposta imediata que se torna associada aos estímulos aversivos. $\mathrm{Na}$ aprendizagem observacional, a expressão facial ou postura de um co-específico torna-se um estímulo condicionado que foi previamente associada com 0 evento aversivo diretamente experienciado e pode agir como um reforçador secundário em situação de aprendizagem futura ${ }^{20}$.

A percepção do estado emocional de outros indivíduos aumenta a probabilidade de exibir comportamento similar e 
de adaptar-se rapidamente aos desafios do ambiente ${ }^{21}$. Essa adaptação social rápida pode ser particularmente importante em situações potencialmente perigosas que induzem a expressão do medo. Assim, é crucial que o indivíduo perceba e responda às características do estímulo ameaçador e da situação em que este se encontra de modo a elaborar uma estratégia de defesa eficiente. Neste caso, os sinais fornecidos pelo comportamento de um co-específico têm um grande valor.

Existem estudos sobre aprendizagem do perigo por meio da experiência com contingências aversivas ${ }^{22}$ ou também sobre a aprendizagem por observação da experiência de outros ${ }^{7,23,24}$. Uma grande parte desses estudos investigou a expressão da resposta condicionada de congelamento a partir da experiência com choques elétricos inescapáveis ou escapáveis. Nesse contexto, foi proposto um modelo de transferência social do medo entre ratos, para caracterizar a interação social durante a qual o medo é transmitido e os efeitos do medo transmitido socialmente ${ }^{25}$.

Esses autores relataram que ratos que observaram e interagiram com outros ratos que passaram pelo condicionamento aversivo de uma resposta de esquiva do choque mostraram facilitação da aprendizagem e memória da resposta de esquiva e exibiram aumento da resposta condicionada de congelamento avaliada no dia seguinte. Contudo, embora haja uma extensa literatura sobre as bases neurais da aprendizagem do medo por experiência direta com contingências aversivas, pouco se sabe sobre os 
mecanismos pelos quais os animais aprendem quando não tiveram a experiência direta do perigo.

É interessante notar que diferentes fontes bibliográficas recentes consideraram a importância de neurônios-espelho no contexto de aprendizagem social e na aprendizagem de respostas de defesa em situações de avaliação dos riscos, perigo e ameaça ${ }^{26}$. Esses neurônios-espelho parecem ser característicos do cérebro de vertebrados que participariam de funções complexas, tal como a compreensão do comportamento de outros ${ }^{27}$. Foi proposto então, que as funções dos neurônios-espelho poderiam também ter importância no contexto de situações que envolvem risco ou ameaça. No contexto de um ataque por predador é importante que a presa seja capaz de discriminar a sua localização e distância, bem como os comportamentos exibidos pelo predador, incluindo vocalização, postura e expressão facial. Mas também pode ser igualmente importante reconhecer a expressão facial e gestos de outro indivíduo que esteja sendo agredido ou ameaçado, identificando as suas emoções ${ }^{26}$.

Diversos trabalhos analisaram o condicionamento clássico aversivo em pombos com associações som-choque e a expressão da resposta de medo condicionado ao contexto1-3,28. Como nos trabalhos com roedores, os trabalhos com pombos demonstram a expressão da resposta de congelamento durante o teste de re-exposição ao contexto. Essa resposta condicionada é considerada como evidência da memória ao contexto aversivo e é expressa com 
uma topografia característica, o congelamento. De um modo geral, quando um pombo recebe um choque elétrico ele apresenta sobressalto, resposta de esvoaçar, comportamentos exploratórios que são então seguidos pela expressão de imobilidade tensa ou congelamento. Quando o choque é precedido por um som, essa sequência é precedida por comportamento exploratório durante o som. Desse modo, considera-se que um pombo que passa pelo treinamento com exposição a som-choque, apresenta um padrão comportamental característico e que pode ser claramente percebido por um pombo que o esteja observando. Assim, o presente trabalho fundamenta-se na consideração de que um pombo (observador) que observe 0 desempenho de um pombo (modelo) durante 0 condicionamento de medo poderá exibir padrão de resposta semelhante à resposta medo condicionado do modelo, quando colocado no contexto em que ocorreu 0 condicionamento. Dessa forma, o objetivo do trabalho foi analisar a aquisição da resposta condicionada de medo por pombos que observaram o treino de condicionamento clássico som-choque em um animal modelo.

\section{MÉTODO}

\section{Amostra}

Foram utilizados 40 pombos, machos, adultos, derivação não controlada da espécie Columba livia, mantidos sob temperatura média de $25^{\circ} \mathrm{C}$ e ciclo-claro escuro $12: 12 \mathrm{~h}$. 
Os procedimentos seguiram o protocolo experimental de respeito aos princípios éticos em experimentação animal aprovado pelo Comitê de Ética em Pesquisa Animal do Instituto de Biologia da UNICAMP (CEUA - Protocolo 2717$1)$.

\section{Procedimento}

Após a chegada ao biotério de aves os animais foram mantidos em quarentena, recebendo banho e tratamento anti-parasitas internos e externos. Durante esse período, os animais foram submetidos a manipulações de caráter dessensibilizatório, profilático (administração de antihelmínticos) e terapêutico (extinção de escabiose). Em seguida foram alojados em gaiolas individuais, com água e comida ad libitum (mistura de quirera de milho, semente de girassol, ração especial para aves, areia e farinha de ostra). Os pombos foram identificados numericamente e catalogados.

O estudo foi constituído pelos seguintes grupos: Grupo Modelo $(n=10)$ : pombos sem acesso à observação de outro, submetidos ao treino com três apresentações de somchoque pareados e que foram testados $24 \mathrm{~h}$ depois ao contexto e $48 \mathrm{~h}$ depois ao som. Grupo Observação $(n=10)$ : pombos com acesso à observação do pombo-modelo durante o treino com três apresentações de som-choque e que foram testados $24 \mathrm{~h}$ depois ao contexto e $48 \mathrm{~h}$ depois ao som.

Inicialmente, os animais do Grupo Modelo foram treinados em condicionamento som-choque, composto por 
03 pareamentos som-choque (aos $5 \mathrm{~min}, 10 \mathrm{~min}$ e $15 \mathrm{~min}$ ), durante uma sessão de treino de 20min (Figura 1A). A sessão de treino foi pareada à sessão de observação de pombos do Grupo Observação, de modo que cada pomboModelo fez par com um pombo observador.

Após o treino som-choque, os pombos foram expostos ao contexto de condicionamento $24 \mathrm{~h}$ após a primeira sessão, e consistiu na colocação do pombo na mesma câmara em que foi realizado o condicionamento clássico com as apresentações de som-choque. A sessão do teste foi realizada com cada pombo individualmente, e teve $20 \mathrm{~min}$ de duração, sem apresentação de qualquer estímulo (Figura 1B).

O teste de condicionamento ao som foi realizado $48 \mathrm{~h}$ após o treino e consistiu na colocação do pombo numa outra câmara com características diferentes daquela em que recebeu as apresentações de som e choque. A sessão do teste teve $10 \mathrm{~min}$ de duração, com apresentação de 03 estímulos sonoros ( $3 \mathrm{~min}, 6 \mathrm{~min}$ e $9 \mathrm{~min}$ ) (Figura 1C). Findo o teste, os animais retornaram ao biotério.

As sessões de treino e teste foram gravadas com sistema digital e o registro do comportamento de congelamento foi baseado na descrição contida no catálogo de comportamentos de pombos ${ }^{23}$, de acordo com a Tabela 1. 
Figura 1. A) Treino de Condicionamento Som-Choque, o Grupo Modelo foi exposto a sessão de 20 minutos com 3 apresentações de pareamento som-choque, os animais do Grupo Observação foram colocados em caixas idênticas, sem apresentação de estímulos; B) O teste ao contexto consistiu na permanência dos animais nas caixas experimentais sem a apresentação de estímulos e C) o teste ao som consistiu em uma sessão de 10 minutos onde ocorreram a apresentação de 3 estímulos sonoros aos 3, 6 e 9 minutos, tanto o Grupo Modelo quanto o Grupo Observação foram expostos aos estímulos.

A Treino de Condicionamento Som-Choque

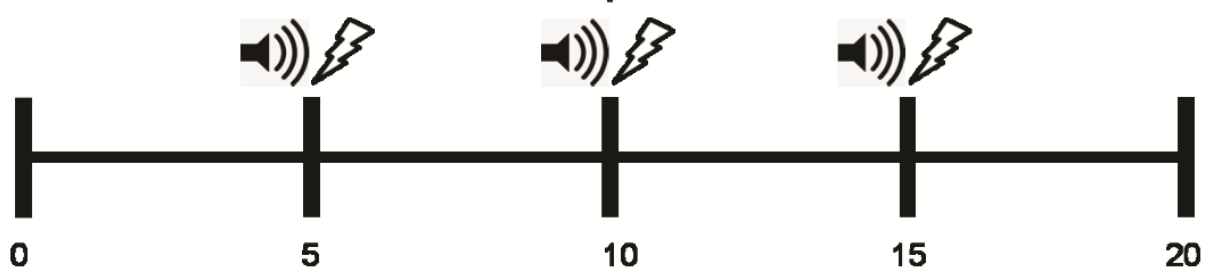

B Teste ao Contexto

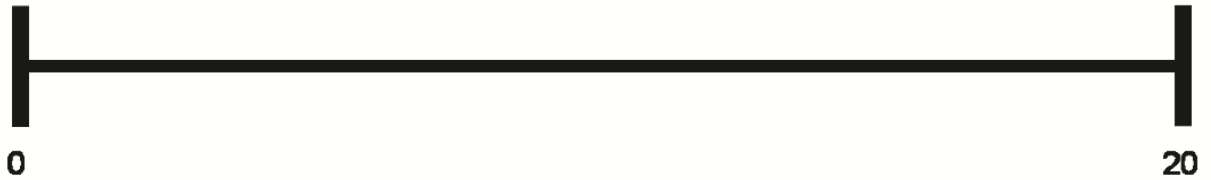

C Teste ao Som

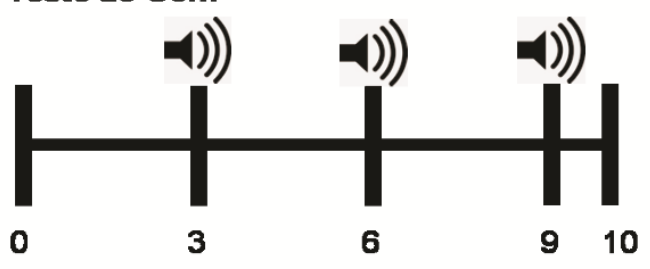

Tabela 1. Descrição contida no catálogo de comportamentos de pombos ${ }^{23}$.

\begin{tabular}{|l|l|}
\hline \multirow{2}{*}{$\begin{array}{l}\text { Congelamento - } \\
\text { freezing. }\end{array}$} & -Flexão total flexão ou parcial das pernas (encolhido); \\
-Separação ampla entre os pés (base de apoio alargada); & -Cauda e asas desalinhadas; \\
-Região ventral ou peitoral do corpo em contato com o piso ou com \\
uma das paredes da câmara; \\
-Pescoço encolhido; \\
-Olhos totalmente abertos; \\
-Respiração acelerada; \\
-Ausência de outros comportamentos observáveis.
\end{tabular}


A fidedignidade dos dados foi avaliada sistematicamente pela análise comparativa de revisão das gravações e das transcrições por dois observadores principal e controle - e a revisão das gravações em vídeo, garantiram o controle da validade e fidedignidade dos dados. Um terceiro observador atuou como controle em das sessões. A transcrição dos dados comportamentais gravados e armazenados em computador fora realizada usando-se o programa EthoLog 2.2 (Ethological Transcription Tool, 1995$\left.99^{29}\right)$. A partir da sequência comportamental obtida foram considerados os comportamentos registrados em blocos de 30 segundos. Foram analisadas as frequências acumuladas em blocos de cinco intervalos de registro, com um total de oito blocos de intervalos de registro para cada sessão.

\section{Análise Estatística}

Os dados relativos à ocorrência de comportamentos foram submetidos a análises estatísticas com o teste ANOVA, tendo grupo como fator e sessões como medida repetida. As análises post hoc para múltiplas comparações foi realizada com o teste Tukey.

\section{RESULTADOS}

Na Figura 2 são apresentados os resultados da ocorrência em segundos de congelamento considerando a sessão (TREINO=T; $C O N T E X T O=C$ e $S O M=S$ ) e o grupo $($ MODELO=M e OBSERVAÇÃO=O). As análises identificaram 
diferenças entre Sessões $\left(F_{5,25}=8,174 ; p<0,0001\right)$. O teste pos-hoc de Tukey identificou diferenças entre as sessões do TM (Sessão de treino do grupo Modelo) e CM (Sessão ao contexto do grupo Modelo; $p<0,05)$. Além disso, foram observadas diferenças entre TO (Sessão de Treino do Grupo Observação) CO e SO (Sessão ao Contexto do Grupo Observação e Sessão ao Som do grupo observação, respectivamente; $\mathrm{p}<0,05)$.

Figura 2. Valores médios+epm de congelamento para as sessões de Treino, Contexto e Som, para os grupos Modelo e Observação.

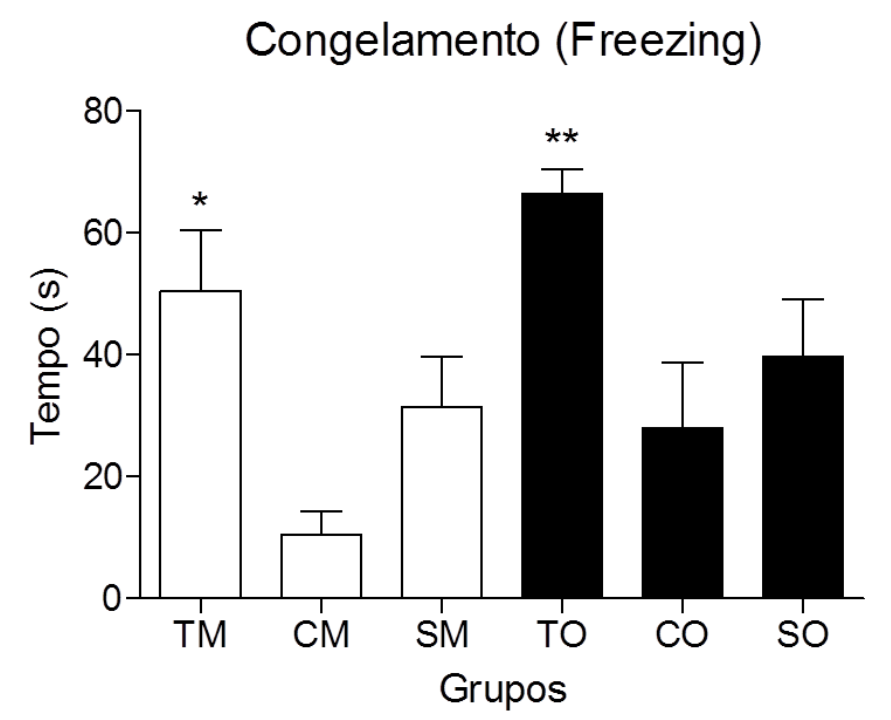

$\mathrm{TM}=$ Treino Grupo Modelo; $\mathrm{CM}=$ Teste ao Contexto do Grupo Modelo; SM = Teste ao Som do Grupo Modelo; TO = Treino Grupo Observação; CO = Teste ao Contexto do Grupo Observação e SO = Teste ao Som do Grupo Observação. * = diferença significante em relação a $\mathrm{CM}$; ** diferença significante em relação a CO e SO.

Na Figura 3 são apresentados os resultados médios da ocorrência de congelamento avaliando-se em blocos de 150 segundos, em A, comparando-se TM com TO; em B 
comparando-se CM com CO e em C, comparando-se SM e SO, é importante notar que em nenhuma das situações a análise de variância ANOVA demonstrou diferença significante.

Figura 3. Ocorrência de Congelamento em blocos de 150 segundos. A) Comparação entre Treino do Grupo Modelo e Treino do Grupo Observação (TM e TO respectivamente); B) Comparação entre Teste ao Contexto Grupo Modelo e Teste ao Contexto do Grupo Observação (CM e CO respectivamente) e C) Comparação entre Teste ao Som do Grupo Modelo e Teste ao Som do Grupo Observação (SM e SO respectivamente). Valores médios+epm. Não houve diferença significante para nenhum dos pontos avaliados.
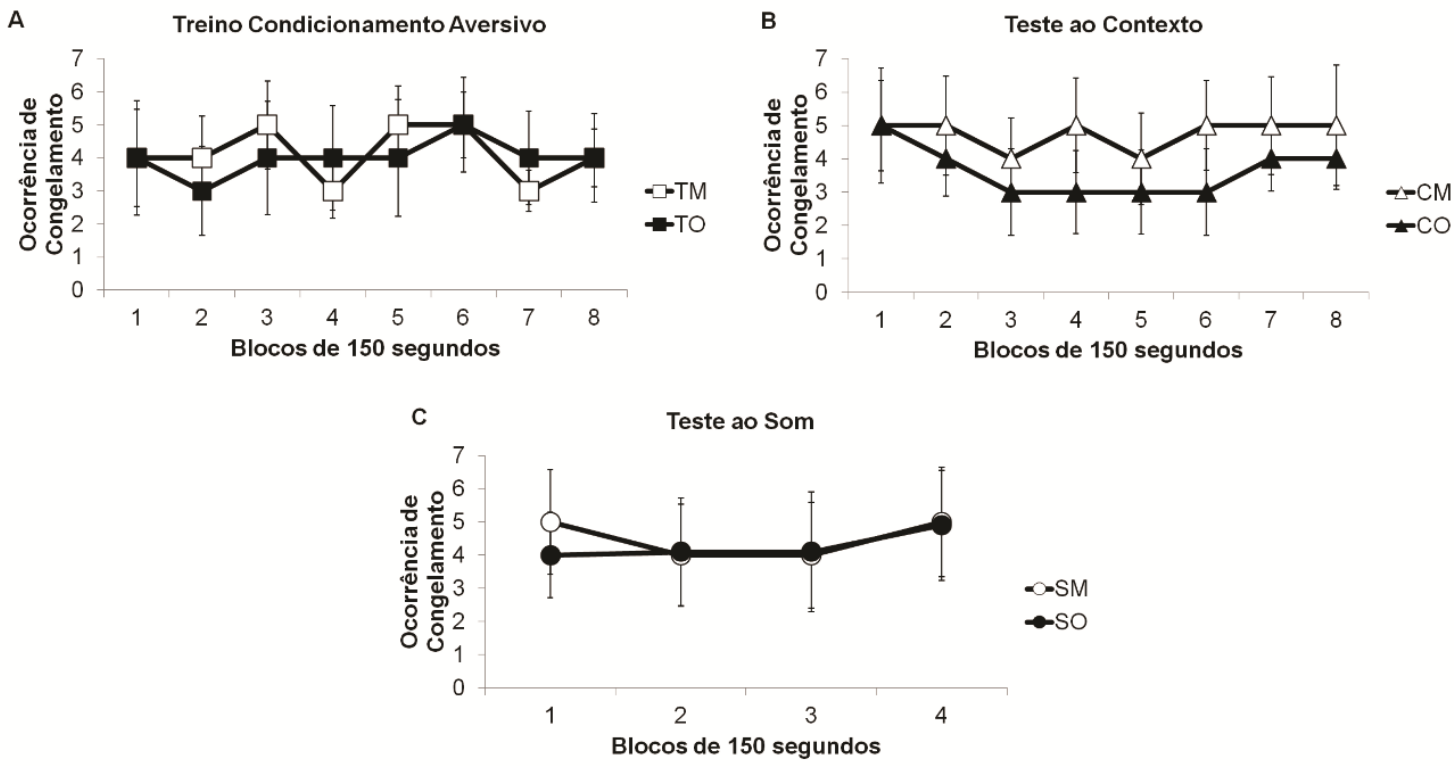

\section{DISCUSSÃO}

O presente estudo apresenta os dados obtidos sobre a aquisição da resposta condicionada do medo por pombos que observaram outro pombo durante um treino de condicionamento clássico, com associações som-choque.

O comportamento de congelamento, ou seja, a cessação do movimento e exibição de imobilidade tensa é 
um comportamento de defesa observado nas situações de perigo quando o ambiente ao qual o animal está exposto não oferece uma rota de fuga ou um esconderijo. A sobrevivência do animal num ambiente perigoso ou ameaçador depende de comportamentos de defesa, entre eles o ataque e a fuga, quando possível. Pesquisadores enfatizaram que é possível estudar as respostas de defesa no laboratório por meio da observação de comportamentos como vigilância, avaliação de risco ou exploração cautelosa e congelamento ${ }^{30}$. A escolha de um determinado comportamento de defesa diante de uma situação de perigo depende do tipo de estímulo ameaçador e do ambiente ao qual o animal está exposto. Os estudos de condicionamento clássico aversivo, usado para a investigação de medo condicionado, usam o comportamento de congelamento como a principal resposta do repertório de defesa que expressa o medo do contexto aversivo/ameaçador. Em nosso presente estudo, observouse a ocorrência de congelamento ${ }^{26}$.

Tanto os animais do Grupo Modelo quanto os animais do Grupo Observação, exibiram durante o treino uma elevada ocorrência de Congelamento. No teste ao contexto, realizado $24 \mathrm{~h}$ após o treino, esta característica se manteve e o mesmo podemos dizer sobre o teste ao som, realizado 48h após o treino, os pombos modelos e observadores apresentaram padrão semelhante de comportamento, apresentando o congelamento ao longo de toda a sessão.

A incidência do comportamento de congelamento, mostra que os animais de ambos os grupos identificaram o 
ambiente ao qual foram expostos como aversivo; pois foi neste ambiente que o Grupo Modelo recebeu o pareamento som-choque. Assim como já foi mencionado, este comportamento é característico de situações aversivas, tal como descrito na literatura ${ }^{31}$.

Assim como os animais modelos, os animais observadores apresentaram elevada ocorrência de congelamento, o que sugere que a experiência de observar outro pombo durante o treino aversivo resultou em comportamentos condicionados de medo, demonstrando que ocorreu aprendizagem de medo por observação. Esse achado corrobora com estudos, que demonstraram que a experiência de observar o comportamento exibidos ou 0 processo de aprendizagem de um co-específico, facilitou a aprendizagem do animal observador ${ }^{8-10}$.

Os dados encontrados no presente estudo evidenciam a aprendizagem do medo por observação em pombos, e favorecem a suposição de que essa aprendizagem possa ser relacionada com os neurônios-espelho. Os neurôniosespelho foram descritos inicialmente em primatas e posteriormente em humanos, como sendo uma classe de neurônios que é ativada quando um indivíduo realiza uma ação ou observa a execução de uma ação por outro, ou seja, a ativação dos neurônios-espelho possibilita a compreensão do significado das ações ou comportamentos, automaticamente ${ }^{32}$. Dessa forma, a aprendizagem por observação evidenciada no presente estudo pode estar 
relacionada com as funções atribuídas aos neurônios-espelho no contexto de situações que envolvem risco ou ameaça ${ }^{26,30}$.

\section{CONCLUSÃO}

O presente trabalho pode estimular novas questões e novas investigações sobre a importância da aprendizagem do medo por observação e de suas possíveis relações com neurônios-espelho.

\section{AGRADECIMENTOS}

Os pesquisadores agradecem à FAPESP pela bolsa de iniciação cientifica. Agradecemos também à Profa. Dra. Elenice Aparecida de Moraes Ferrari († In Memmorian), pela confiança, dedicação e todo conhecimento compartilhado.

\section{REFERÊNCIAS}

1.Faria RS, Sartori CR, Canova F, Ferrari EAM. Classical Aversive Conditioning induces increased expression of mature-BDNF in the hippocampus and amygdala of pigeons. Neuroscience 2013;255:12233. http://dx.doi.org/10.1016/j.neuroscience.2013.09.054

2. Macêdo-Souza C. Condicionamento Clássico: A Ansiedade Patológica e um Modelo Animal de Ansiedade. Curitiba: Editora Appris, 2021. 3.Canova F, Faria RS. Proteína Zenk e o Condicionamento Clássico do Medo. Neurociências 2014;10:37-48. https://www.researchgate.net/publication/263542050 Neurociencias Volume $10 \mathrm{~N} 1$ janeiromarco de 2014 Proteina Zenk e o condi cionamento classico do medo/link/0a85e53b2eccb4603b000000/do wnload

4.Nicol CJ. Development, direction, and damage limitation: social learning in domestic fowl. Learn Behav 2004;32:72-81. http://dx.doi.org/10.3758/BF03196008

5. Laland KN, Toyokawa W, Oudman T. Animal learning as a source of developmental bias. Evol Devel 2020;22:126-42. https://doi.org/10.1111/ede.12311

6. Nicol CJ. How animals learn from each other. App Anim Behav Sci 2006;100:58-63. https://doi.org/10.1016/j.applanim.2006.04.004 7.Olsson A, Phelps EA. Social learning of fear. Nat Neurosci 2007;10:1095-102. https://doi.org/10.1038/nn1968 
8. Biederman GB, Robertson HA, Vanayan M. Observational learning of two visual discriminations by pigeons: a within-subjects design. J Exp Anal Behav 1986;46:45-9. http://dx.doi.org/10.1901/jeab.1986.4645

9.Klein ED, Zentall TR. Imitation and Affordance Learning by Pigeons (Columba livia). J Comp Psychol 2003;117:414-9. https://doi.org/10.1037/0735-7036.117.4.414

10. Howard ML, White GK. Social influence in pigeons (Columba livia): the role of differential reinforcement. J Exp Anal Behav 2003;79:17591. http://dx.doi.org/10.1901/jeab.2003.79-175

11.Johnston ANB, Burne THJ, Rose SP. Observation learning in day old chicks using a one trial passive avoidance learning paradigm. Ani Behav 1998;56:347-53. http://dx.doi.org/10.1006/anbe.1998.0901

12.Rosa-Salva O, Daisley JN, Regolin L, Vallortigara G. Lateralization of social learning in the domestic chick, Gallus gallus domesticus: learning to avoid. Ani Behav 2009;78:847-56. https://doi.org/10.1016/j.anbehav.2009.06.021

13.Rogers LJ. Brain Lateralization and Cognitive Capacity. Animals 2021;11:1996. https://doi.org/10.3390/ani11071996

14.Rosa-Salva O, Daisley JN, Regolin L, Vallortigara G. Timedependent lateralization of social learning in the domestic chick (Gallus gallus domesticus): Effects of retention delays in the observed lateralization pattern. Behav Brain Res 2010;212:152-8. http://dx.doi.org/10.1016/j.bbr.2010.04.004

15. Marler P. Birdsong and speech development: could there be parallels? Am Sci 1970;58:669-73.

16. Beecher MD, Akçay Ç. Social factors in bird-song development: Learning to sing with friends and rivals. Learn Behav 2021;49:137-49. http://dx.doi.org/10.3758/s13420-020-00441-6

17. Nottebohm F. Ontogeny of bird song. Science 1970;167:950-6. https://www.jstor.org/stable/1728208

18. Baptista LF, Petrinovich L. Social interaction, sensitive phases and the song template hypothesis in the white-crowned sparrow. Ani Behav 1984;32:175-81. https://doi.org/10.1016/S0003-3472(84)80335-8

19.Kramar CP, Castillo-Díaz F, Gigante ED, Medina JH, Barbano MF. The late consolidation of an aversive memory is promoted by VTA dopamine release in the dorsal hippocampus. Eur J Neurosci 2021;53:841-51. https://doi.org/10.1111/ejn.15076 20. Hatfield E, Cacioppe JT, Rapson RL. Emotional contagion. New York: Cambridge University Press; 1993, 252p. https://doi.org/10.1111/1467-8721.ep10770953

21.Maren S. Neurobiology of Pavlovian fear conditioning. Annu Rev Neurosci 2001;24:897-931.

http://dx.doi.org/10.1146/annurev.neuro.24.1.897

22. Church RM. Emotional Reactions of Rats to the Pain of Others. J Comp Physiol Psychol 1959;52:132-4. http://dx.doi.org/10.1037/h0043531

23.Langford DJ, Crager SE, Shehzad Z, Smith SB, Sotocinal SG, Levenstadt JS, et al. Social modulation of pain as evidence for empathy 
in

mice.

Science

$2006 ; 312: 1967-70$.

http://dx.doi.org/10.1126/science.1128322

24.Knapska E, Mikosz M, Werka T, Maren S. Social Modulation of learning in rats. Learn Mem 2009;30;17:35-42. http://dx.doi.org/10.1101/Im.1670910

25.Blanchard DC, Griebel G, Pobbe R, Blanchard RJ. Risk assessment as an evolved threat detection and analysis process. Neurosci Biobehav Rev

2011;35:991-8.

http://dx.doi.org/10.1016/j.neubiorev.2010.10.016

26.Canova F, Pelaquim A, Ferrari EAM. Memória Aversiva em Mamíferos e Aves: Relação entre o Hipocampo e o Condicionamento Clássico Aversivo. J Health Sci 2015;17:301-6. https://doi.org/10.17921/2447-8938.2015v17n4p\%25p

27.Reis F, Schenka AA, Melo LL, Ferrari EA. Role of the hippocampus in contextual memory after classical aversive conditioning in pigeons (C. livia). Braz J Med Biol Res 1999;32:1127-31. https://doi.org/10.1590/S0100-879X1999000900012

28. Miller G. Neuroscience. Mirror neurons may help songbirds stay in tune. Science 2008;319:269.

http://dx.doi.org/10.1126/science.319.5861.269a

29.Ottoni EB. EthoLog 2.2: a tool for the transcription and timing of behavior observation sessions. Behav Res Meth 2000;32:446-9. http://dx.doi.org/10.3758/BF0320814

30.Blanchard DC, Blanchard RJ. Ethoexperimental approaches to the biology of emotion. Annu Rev Psychol 1988;39:43-68. http://dx.doi.org/10.1146/annurev.ps.39.020188.000355

31.Valentinuzzi VS, Ferrari EAM. Habituation to Sound During Morning and Night Sessions in Pigeons (Columba livia). Phys Behav 1997;62:1203-9. https://doi.org/10.1016/S0031-9384(97)00009-7

32.Cattaneo L, Rizzolatti G. The Mirror Neuron System. Arch Neurol 2009;66:557-60. http://dx.doi.org/10.1001/archneurol.2009.41 